\title{
Self-Consistent Tip Conditioning for Tip-Enhanced Raman Spectroscopy in an Ambient Environment
}

\author{
Yasuyuki Yokota, ${ }^{1,2, *}$ Misun Hong, ${ }^{1}$ Norihiko Hayazawa, ${ }^{1}$ Bo Yang,,${ }^{1 \dagger}$ Emiko Kazuma, ${ }^{1,2}$ and \\ Yousoo $\mathrm{Kim}^{1, *}$
}

${ }^{1}$ Surface and Interface Science Laboratory, RIKEN, 2-1 Hirosawa, Wako, Saitama 351-0198, Japan

2 JST PRESTO, 4-1-8 Honcho, Kawaguchi, Saitama 332-0012, Japan

${ }^{\dagger}$ Present address: School of Science, Xijing University, 1 Xijing Road, Xi'an, Shaanxi 710123, China.

- Preparation of Samples for SERS Measurements

- Calculation of Vibrational Spectra

- Figure S1: Schematic of the optical setup

- Figure S2: Comparison of TERS, SERS, and DFT simulated spectra

- Figure S3: Normalized STML spectra of $\mathrm{Au}(111)$ sorted in measurement order

- Figure S4: Position dependent TERS spectra obtained inside and outside of a hole

- Figure S5: SEM images of the Au tips obtained before and after control experiments 


\section{Preparation of Samples for SERS Measurements}

SERS active Au wires $(0.25 \mathrm{~mm}$ diameter) were prepared by 40 oxidation/reduction cycles in a $0.1 \mathrm{M} \mathrm{KCl}$ solution. The potential was consecutively swept between -0.2 and $1.2 \mathrm{~V} \mathrm{vs.} \mathrm{Ag} / \mathrm{AgCl}(3.0 \mathrm{M} \mathrm{NaCl})$ at $0.1 \mathrm{~V} / \mathrm{s}$ using a potentiostat (HZ7000, Hokuto denko). After rinsing with Milli-Q water, $\mathrm{Ph}_{3} \mathrm{SH}$ SAMs were fabricated on the activated $\mathrm{Au}$ wires in a similar manner as the SAMs on $\mathrm{Au}(111)$. SERS spectra in air were obtained under the experimental conditions similar to TERS (Figure S1).

\section{Calculation of Vibrational Spectra}

The optimized structures and vibrational spectra of $\mathrm{Ph}_{3} \mathrm{~S}-\mathrm{Au}_{2}$ were calculated using Gaussian $16 .{ }^{\mathrm{S} 1}$ Details of the simulation are published elsewhere. ${ }^{\mathrm{S} 2}$ Briefly, density functional theory (DFT) calculations using the B3LYP hybrid functional were performed initially to optimize the structure and obtain the vibrational frequencies. ${ }^{\mathrm{S} 3}$ In the computation, triple- $\zeta$ valencepolarization basis sets $\left(\right.$ Def2-TZVP ${ }^{\mathrm{S} 4}$ ) are utilized for the molecule whereas Au atoms are described with the Def-SVP basis set $^{\mathrm{S} 5}$ and effective core potentials ${ }^{\mathrm{S} 6}$. The Raman scattering strength was calculated by the static polarizability and convoluted using Lorentzian broadening with a full width at half maximum (FWHM) of $16 \mathrm{~cm}^{-1}$. To correct anharmonicity effects, the wavenumbers were scaled by a factor of 0.98 . 


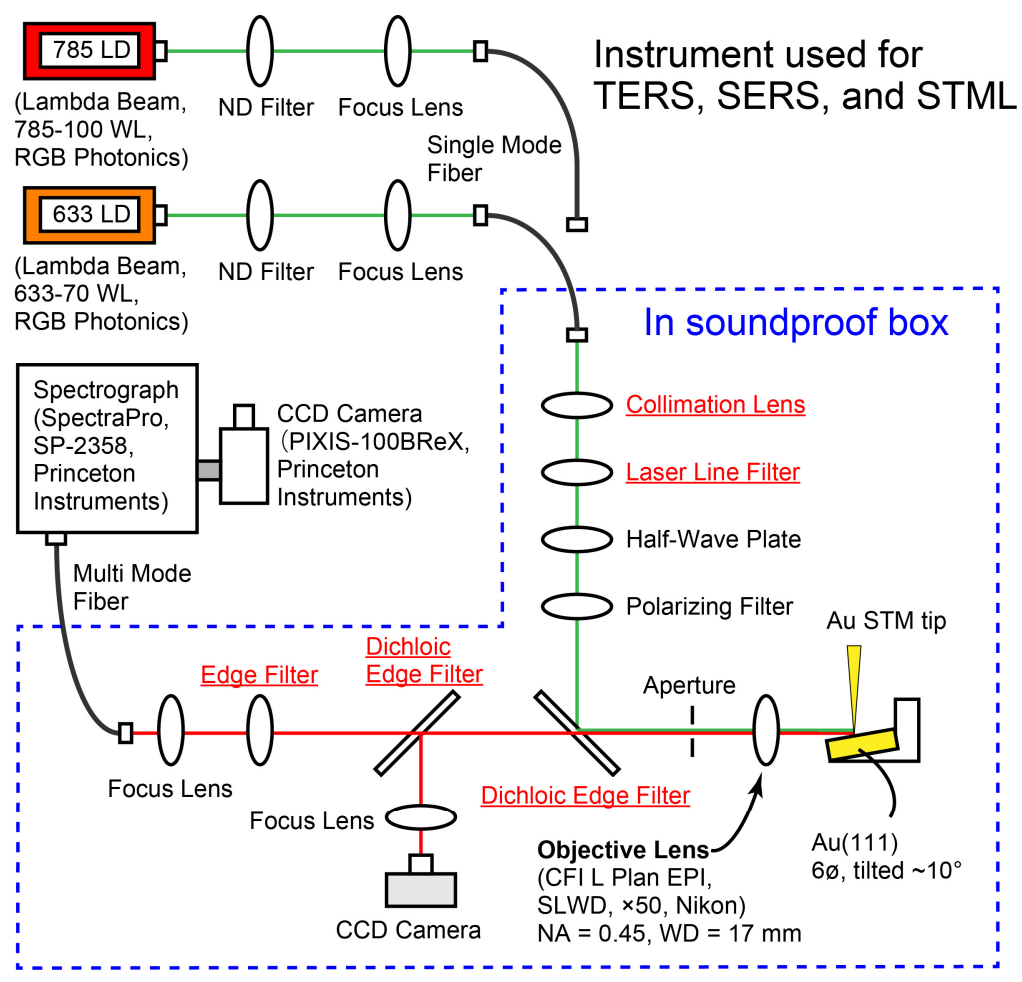

Figure S1. Schematic of the optical setup for TERS, SERS, and STML measurements. The laser diodes, including lenses and filters underlined, can be changed within 5 min while maintaining the same STM scanning area (see Figure 5(a)-(c)). STML measurements were performed with the same photon detection system as TERS and SERS except for the use of a 150 grooves/mm grating. 

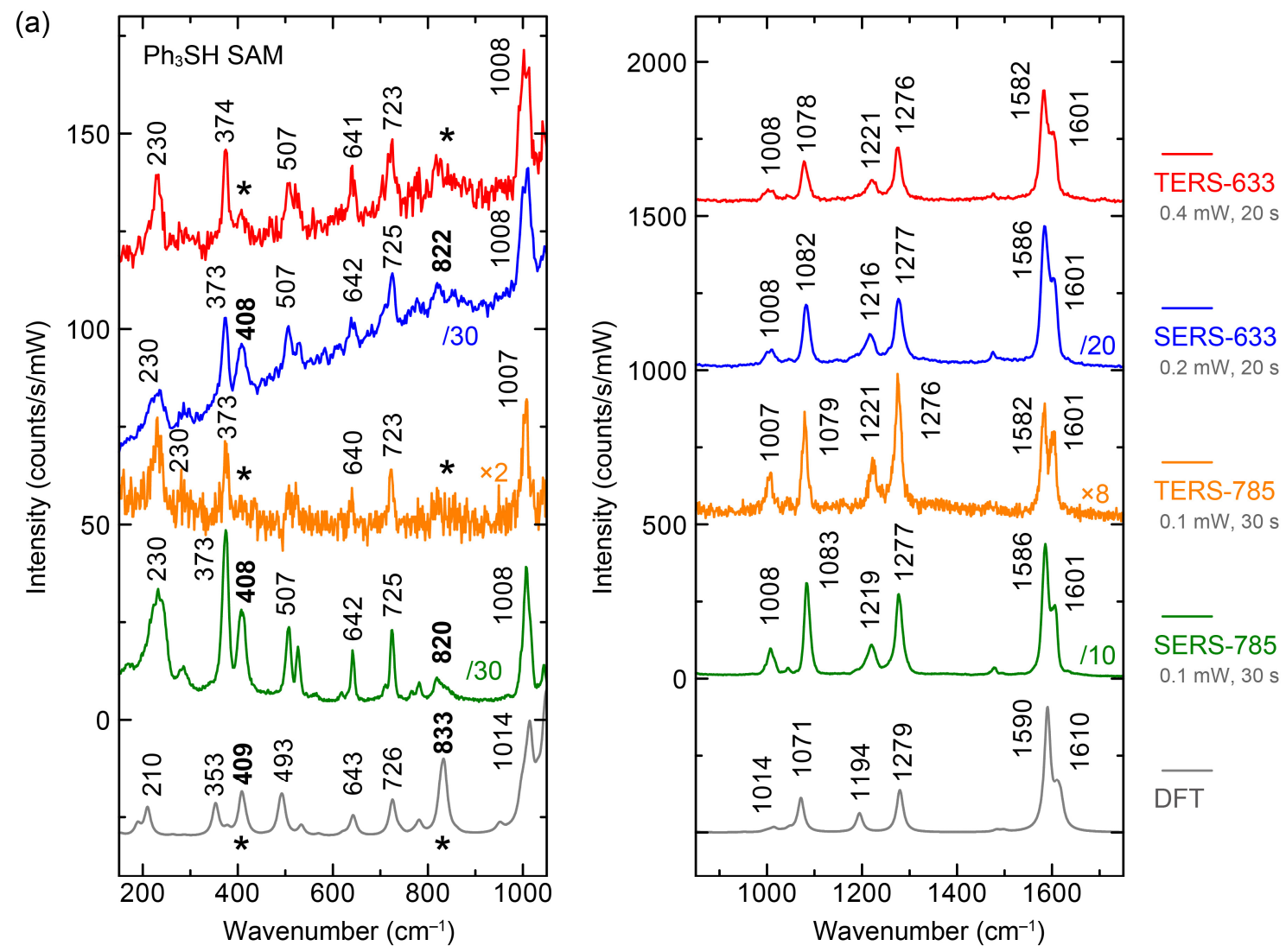

(b)
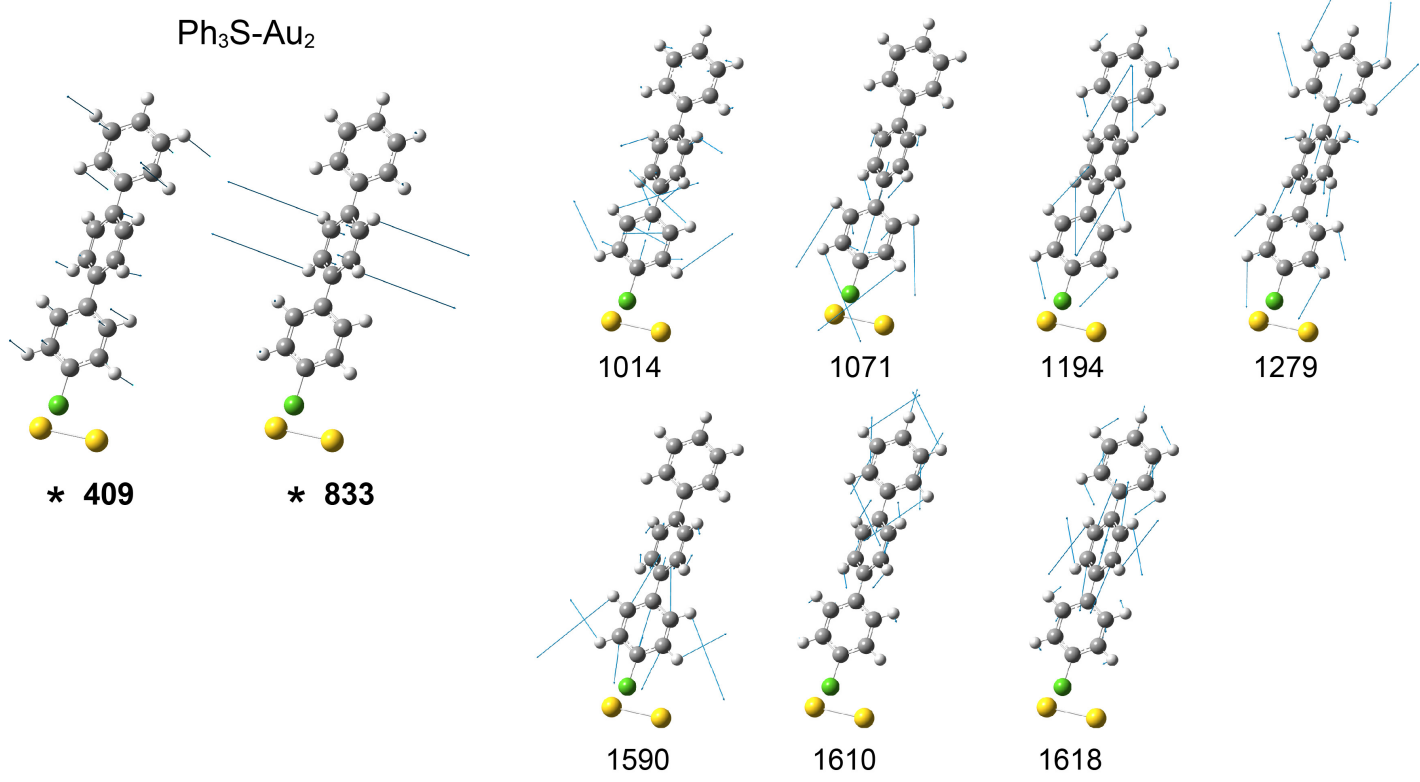

Figure S2. Comparison of TERS and SERS spectra of $\mathrm{Ph}_{3} \mathrm{SH}$ SAMs and calculated Raman spectra of $\mathrm{Ph}_{3} \mathrm{~S}-\mathrm{Au}_{2}$ cluster.

(a) The calculated spectra were scaled to the corresponding experimental spectra. To correct for anharmonicity effects, the wavenumbers of the calculated spectrum were scaled by a factor of 0.98. (b) Geometrically optimized structures and characteristic vibration modes of the $\mathrm{Ph}_{3} \mathrm{~S}-\mathrm{Au}_{2}$ cluster are displayed using Gauss View software. ${ }^{\mathrm{S} 7}$ Note that the asterisk vibrational modes, which possess displacement vectors perpendicular to the long axis of $\mathrm{Ph}_{3} \mathrm{SH}$ molecule, were observed very weakly in the TERS measurements irrespective of the excitation wavelength. Corresponding SERS peaks were also weaker than DFT simulated peaks. These observations indicate the clear electric-field direction dependences of each Raman spectroscopy. ${ }^{\mathrm{S} 8}$ 


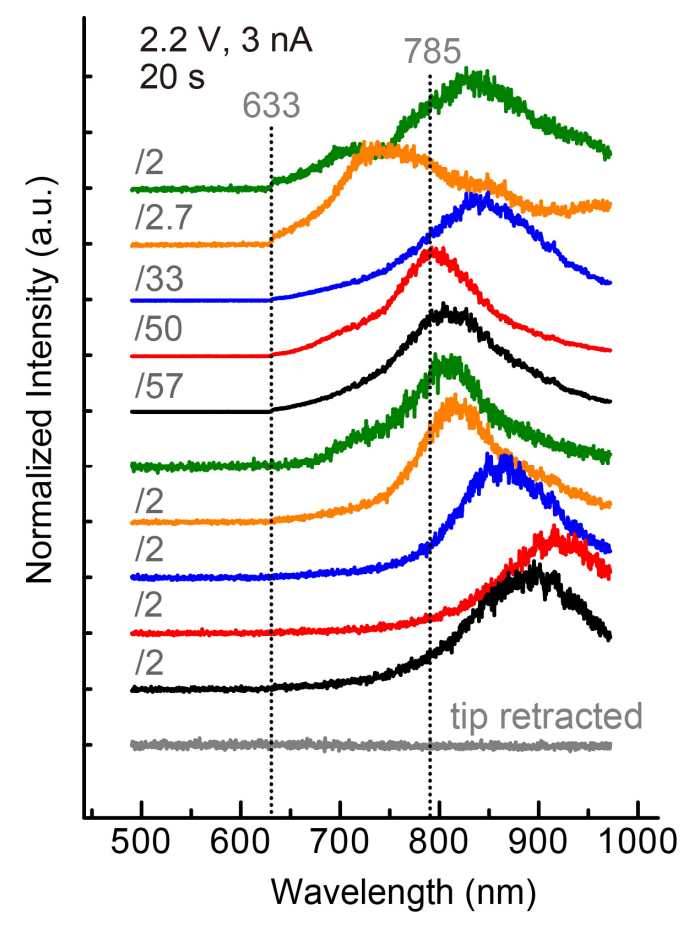

Figure S3. Normalized STML spectra of Au(111) sorted in measurement order. In addition to the tendency for the peak to shift from the short to the long-wavelength side, exceptional behavior was also observed. This would be derived from the sudden changes of the tip and/or the sample owing to the applied high bias voltage. Notably, several measurements were performed between each plot. 

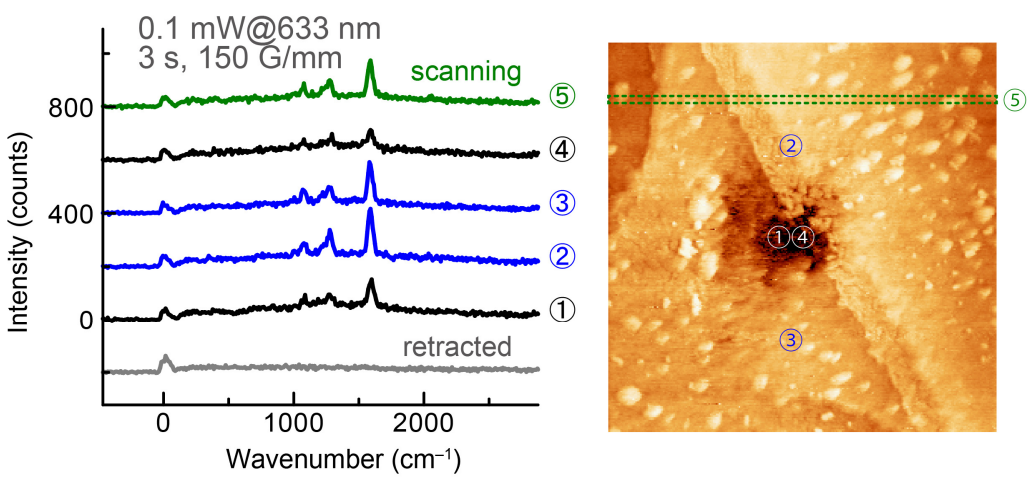

Figure S4. Position dependence of TERS spectra. The STM image shown on the right is the same as Figure 5(b). $V_{\mathrm{s}}=0.4$ $\mathrm{V}, I=0.4 \mathrm{nA}$. Z-range of the STM image is $1.2 \mathrm{~nm}$. The TERS spectrum of \#5 was obtained during the STM scan indicated by the area in the STM image. 

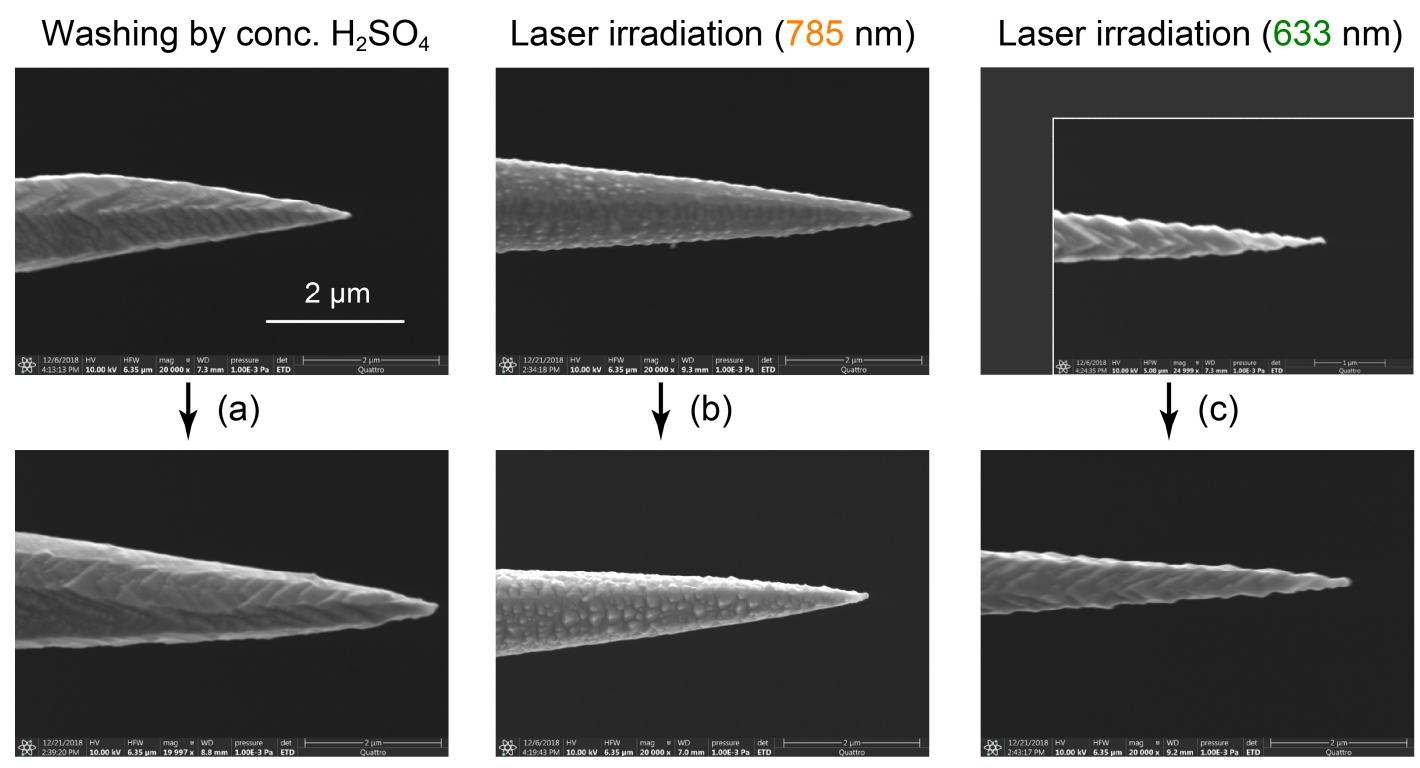

TERS (785 nm)

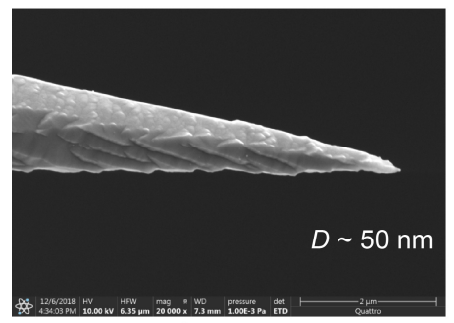

$\downarrow$ (d)

TERS $(633 \mathrm{~nm})$

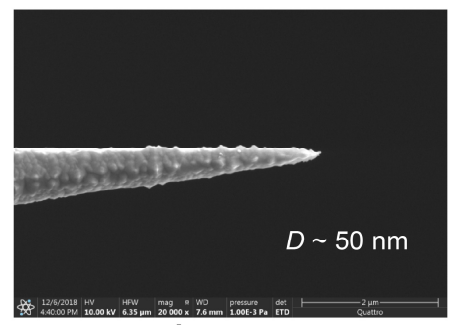

$\downarrow$ (e)
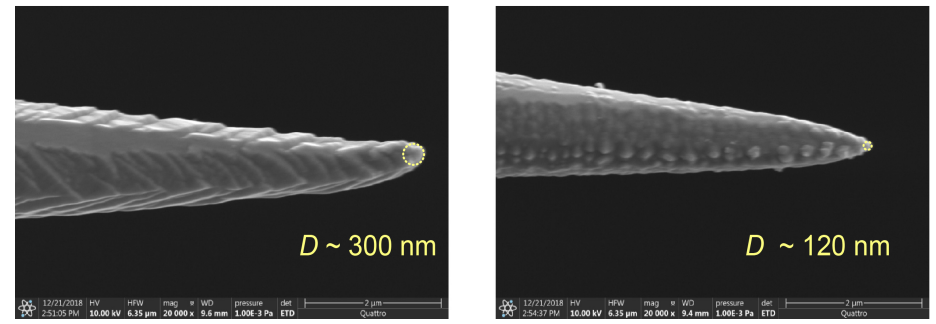

Figure S5. Comparison of SEM images taken before and after control experiments. (a) Just washed with conc. $\mathrm{H}_{2} \mathrm{SO}_{4}$ and milli-Q water, (b)(c) the tip apex was irradiated by (b) 785 and (c) $633 \mathrm{~nm}$ laser at the power of $0.1 \mathrm{~mW}$ for $1 \mathrm{~h}$ in addition to the process in (a), (d)(e) TERS measurements $\left(V_{\mathrm{s}}=0.4 \mathrm{~V}, I=0.4 \mathrm{nA}\right)$ of $\mathrm{Ph}_{3} \mathrm{SH}$ SAMs on Au(111) at (d) $785 \mathrm{~nm}$ with 0.1 $\mathrm{mW}$ and (e) $633 \mathrm{~nm}$ with $0.1 \mathrm{~mW}$ for less than $8 \mathrm{~min}$ in addition to the process in (a). 


\section{$\underline{\text { References }}$}

S1. Frisch, M. J.; Trucks, G. W.; Schlegel, H. B.; Scuseria, G. E.; Robb, M. A.; Cheeseman, J. R.; Scalmani, G.; Barone, V.; Petersson, G. A.; Nakatsuji, H.; et al. Gaussian 16 Rev. A.03, Gaussian, Inc.: Wallingford, CT, 2016.

S2. Saikin, S. K.; Olivares-Amaya, R.; Rappoport, D.; Stopa, M.; Aspuru-Guzik, A. On the Chemical Bonding Effects in the Raman Response: Benzenethiol Adsorbed on Silver Clusters. Phys. Chem. Chem. Phys. 2009, 11, 9401-9411.

S3. Stephens, P. J.; Devlin, F. J.; Chabalowski, C. F.; Frisch, M. J. Ab Initio Calculation of Vibrational Absorption and Circular Dichroism Spectra Using Density Functional Force Fields. J. Phys. Chem. 1994, 98, 11623-11627.

S4. Weigend, F.; Ahlrichs, R. Balanced Basis Sets of Split Valence, Triple Zeta Valence and Quadruple Zeta Valence Quality for H to Rn: Design and Assessment of Accuracy. Phys. Chem. Chem. Phys. 2005, 7, 3297-3305.

S5. Schäfer, A.; Horn, H.; Ahlrichs, R. Fully Optimized Contracted Gaussian Basis Sets for Atoms Li to Kr. J. Chem. Phys. 1992, 97, 2571-2577.

S6. Andrae, D.; Häußermann, U.; Dolg, M.; Stoll, H.; Preuß, H. Energy-Adjusted Ab Initio Pseudopotentials for the Second and Third Row Transition Elements. Theor. Chim. Acta 1990, 77, 123-141.

S7. Dennington, R.; Keith, T. A.; Millam, J. M. Gaussview, Ver. 6, Semichem Inc., Shawnee Mission, KS, 2016.

S8. Hong, M.; Yokota, Y.; Hayazawa, N.; Kazuma, E.; Kim, Y. Homogeneous Dispersion of Aromatic Thiolates in the Binary Self-Assembled Monolayer on Au(111) Via Displacement Revealed by Tip-Enhanced Raman Spectroscopy. J. Phys. Chem. C 2020, 124, 13141-13149. 